\title{
The roles of ram-pressure stripping and minor mergers in the evolution of galaxies
}

\author{
Takashi Okamoto ${ }^{1,2}$ and Masahiro Nagashima ${ }^{3,1}$ \\ ${ }^{1}$ Department of Physics, University of Durham, South Road, Durham, DH1 3LE UK \\ email: takashi.okamoto@durham.ac.uk \\ ${ }^{2}$ NAOJ, NINIS, Mitaka, Tokyo 181-8588 Japan \\ ${ }^{3}$ Department of Physics, Kyoto University, Kyoto 606-8502, Japan
}

\begin{abstract}
We investigate environmental effects on evolution of bright cluster galaxies in a $\Lambda$ dominated cold dark matter universe using a combination of dissipationless $N$-body simulations and a semi-analytic galaxy formation model. We incorporate effects of ram-pressure stripping (RPS) and minor merger-induced small starburst (minor burst) into our model. By considering minor burst, the observed morphology-radius relation is successfully reproduced. When we do not consider minor burst, the RPS hardly increases the intermediate B/T population. In addition, the RPS and minor burst are not important for colours or star formation rates of galaxies in the cluster core if star formation time-scale is properly chosen, because the star formation is sufficiently suppressed by consumption of the cold gas. We also find that SF in bulge-dominated galaxies is mainly terminated by starburst induced by major mergers in all environments.
\end{abstract}

\section{Introduction}

It has been found that galaxy morphology is a function of environment (Dressler 1980; Whitmore et al. 1993) and redshift (Dressler et al. 1997). Colours and star formation rate (SFRs) of galaxies also show similar dependence on environment and redshift (e.g. Butcher \& Oemler 1984; Lewis et al. 2002). To account for these observational trends, several mechanisms that may suppress the star formation (SF) and transform one morphological type into another have been proposed.

Interaction between galaxies is one possible process to promote morphological transformation. Numerical simulations confirmed that major mergers produce galaxies resembling ellipticals as merger remnants (Barnes 1996) and that accretion of small satellites onto their host spiral lead a host spiral to S0 type (Walker et al. 1996). Because the galaxy merger triggers starbursts, the cold gas contained in original galaxies is exhausted in a very short time.

The second mechanism is removal of hot gas reservoir. In denser environments such as clusters, diffuse hot gas reservoir that is originally confined in haloes of non-central galaxies becomes part of the intracluster medium. A galaxy, whose hot gas reservoir is slowly removed, exhausts its cold gas in its SF time-scale (strangulation).

The above two processes, i.e. major merger-induced starburst and strangulation, has been incorporated in most of semi-analytic (SA) models which have failed to reproduce intermediate $B / T$ population (Okamoto \& Nagashima 2001; Diaferio et al. 2001). In this paper, we introduce two additional processes in order to solve this problem. One is rampressure stripping (RPS) of the cold gas from galactic disks (Gunn \& Gott 1972). Another is minor merger-induced small starburst. Hydrodynamic simulations showed that some fraction of disk gas is driven to the galactic centre by a minor merger (Walker et al. 1996). By using a combination of $N$-body simulations with a SA model, we study the roles of 
the above four processes in the evolution of galaxies and what process is responsible for the formation of intermediate $B / T$ galaxies.

\section{Models}

Here we consider a $\Lambda$-dominated cold dark matter universe $\left(\Omega_{0}=0.3, \lambda_{0}=0.7, \Omega_{b}=\right.$ $0.015 h^{-2}, H_{0}=100 h \mathrm{~km} \mathrm{~s}^{-1} \mathrm{Mpc}^{-1}, h=0.7$, and $\left.\sigma_{8}=1\right)$. We simulate a cluster region to study cluster galaxies and a region whose density is almost the same as the mean density of the universe to study field population. We then construct merger trees of virialised haloes and their substructure haloes. The details of this procedure is given in Okamoto \& Habe $(1999,2000)$.

To clarify the effect of each physical process, here we examine four models. The model in which we consider neither the RPS nor the minor burst is called the standard model, because this is the standard prescription in SA models. Model parameters in the standard model are normalised so that they reproduce luminosity functions, cold gas fractions, and sizes of field galaxies. We also show the model using four times longer SF time-scale than the standard model to see the effect of the strangulation. This model is referred to as the low SFR model. The model with the minor burst and the model with the RPS are called "minor burst" and "RPS", respectively. In these models, we use the same parameters as used in the standard model. The details can be found in Okamoto \& Nagashima (2003).

\section{Results}

In Fig. 1, we show colour-magnitude relations (CMRs) for simulated galaxies in the standard field model and cluster cores $\left(r<0.5 h^{-1} \mathrm{Mpc}\right)$. We classify galaxies with $B$ band $B / T<0.4$ as late-type and others as early-type galaxies. First of all, the CMRs for early-type galaxies are quite similar in all models, even in the field. It suggests that major merger-induced starbursts mainly truncate SF in early-type galaxies. On the other hand, colours of late-type galaxies are sensitive to the SF time-scale and their environments. Blue population seen in the field model is vanished in the cluster if we use appropriate SF time-scales. Since, in the low SFR model, late-type galaxies have much bluer colours than those in the standard model, it can be said that strangulation is the process that stops SF of late-type galaxies in cluster cores. The suppression of SF by strangulation is so efficient that the effect of the RPS is hardly seen except in the low SFR + RPS model.

It has been known that galaxies in the inner regions of clusters are redder than those in the outer regions. Recent observations have confirmed the existence of colour gradients in the galaxy population, which appear to continue even beyond the virial radius (e.g. Carlberg et al. 1997). In the left panels of Fig. 2 we show the $B-V$ colours of the cluster galaxies at $z=0.2$ as a function of projected radius. The projected radius is normalised by $R_{200}$ that is the radius inside which the mean density is 200 times the critical density of the universe at a given redshift. The observational data are taken from Diaferio et al. (2001).

For the standard and minor burst models, the effect of the RPS can be seen only at large radii, because, in the central part, the $\mathrm{SF}$ is strongly suppressed by the strangulation. By comparing the low SFR + RPS model with the low SFR model, it is found that the RPS effectively suppresses SF at $r<R_{200}$ if there is some cold gas left in the galaxies. While the minor burst makes galaxies slightly redder than those in the standard model, the models with and without the minor burst produces almost the same results.

Recently, some authors have studied the morphology-density (or radius) relations by using a hybrid method of $N$-body simulations and SA models (Okamoto \& Nagashima 


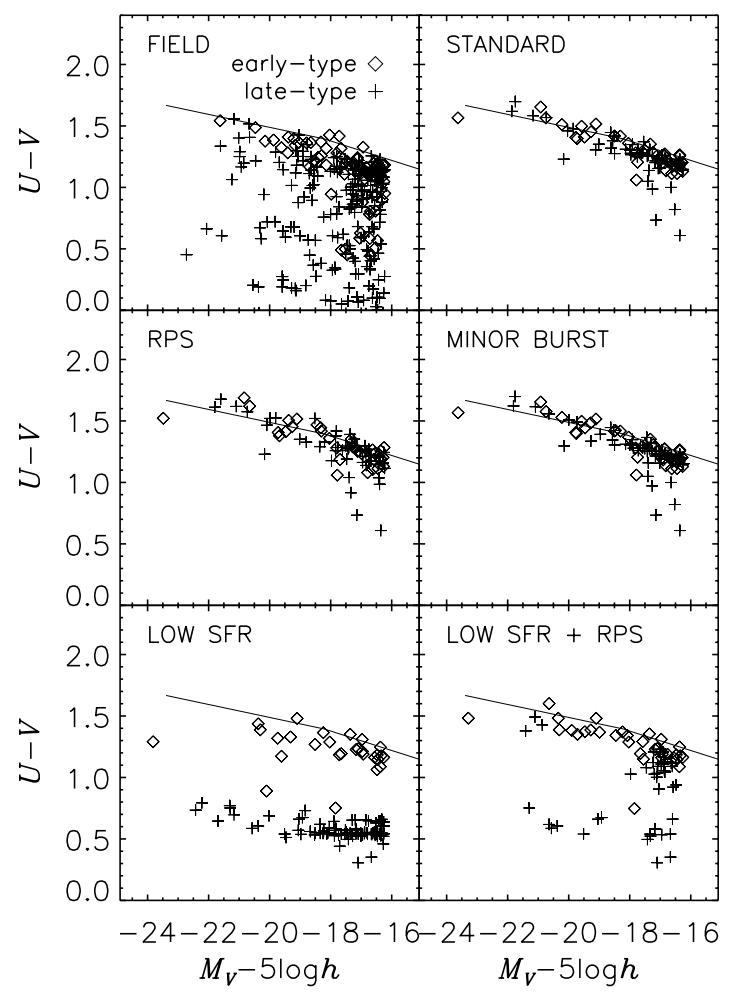

Figure 1. CMRs at $z=0$. We show $U-V$ colours of galaxies in the field model and galaxies within the cluster core in the standard, RPS, minor burst, low SFR, and low SFR + RPS models as a function of $V$-band luminosity. Early-type galaxies and late-type galaxies are represented by diamonds and crosses, respectively. The solid lines show the observed CMR for cluster ellipticals by Bower et al. (1992).

2001; Diaferio et al. 2001; Springel et al. 2001). In their work, S0 or intermediate $B / T$ population in clusters is hardly reproduced unless a wider $B / T$ range is adopted to classify simulated galaxies as S0s. Here we examine how the RPS and minor burst affect the intermediate $B / T$ population in clusters.

We show morphological fractions in the cluster as a function of radius in the righ panels of Fig. 2. The simulated galaxies are classified according to their $B$-band $B / T ; B / T>0.7$ as bulge-dominated, $B / T<0.4$ as disk-dominated, and $0.4<B / T<0.7$ as intermediate. By comparison of the CNOC sample with the standard model, it is confirmed that the intermediate fraction in the standard model is much smaller than the observation, while the bulge-dominated fraction is reproduced. Even when we consider the RPS, the intermediate fraction is still too small. It is because most of the disk-dominated galaxies are almost pure disk galaxies without minor bursts (Okamoto \& Nagashima 2003), and then the RPS simply darkens the disk-dominated galaxies rather than increasing their $B / T$ s. The strange behaviour seen in the RPS models at $r / R_{200}>0.7$ results from the small number of galaxies at these radii because of the spherical high-resolution region.

The minor bursts increases the intermediate fraction without changing the bulgedominated fraction because it affects only galaxies with small $B / T$ s. As a result, the minor burst model reproduces the observed fractions quite well despite the fact that it does not influence other properties of the galaxies. Thanks to minor bursts, now the disk-dominated galaxies tend to be non-pure disk galaxies. Consequently, the RPS can 

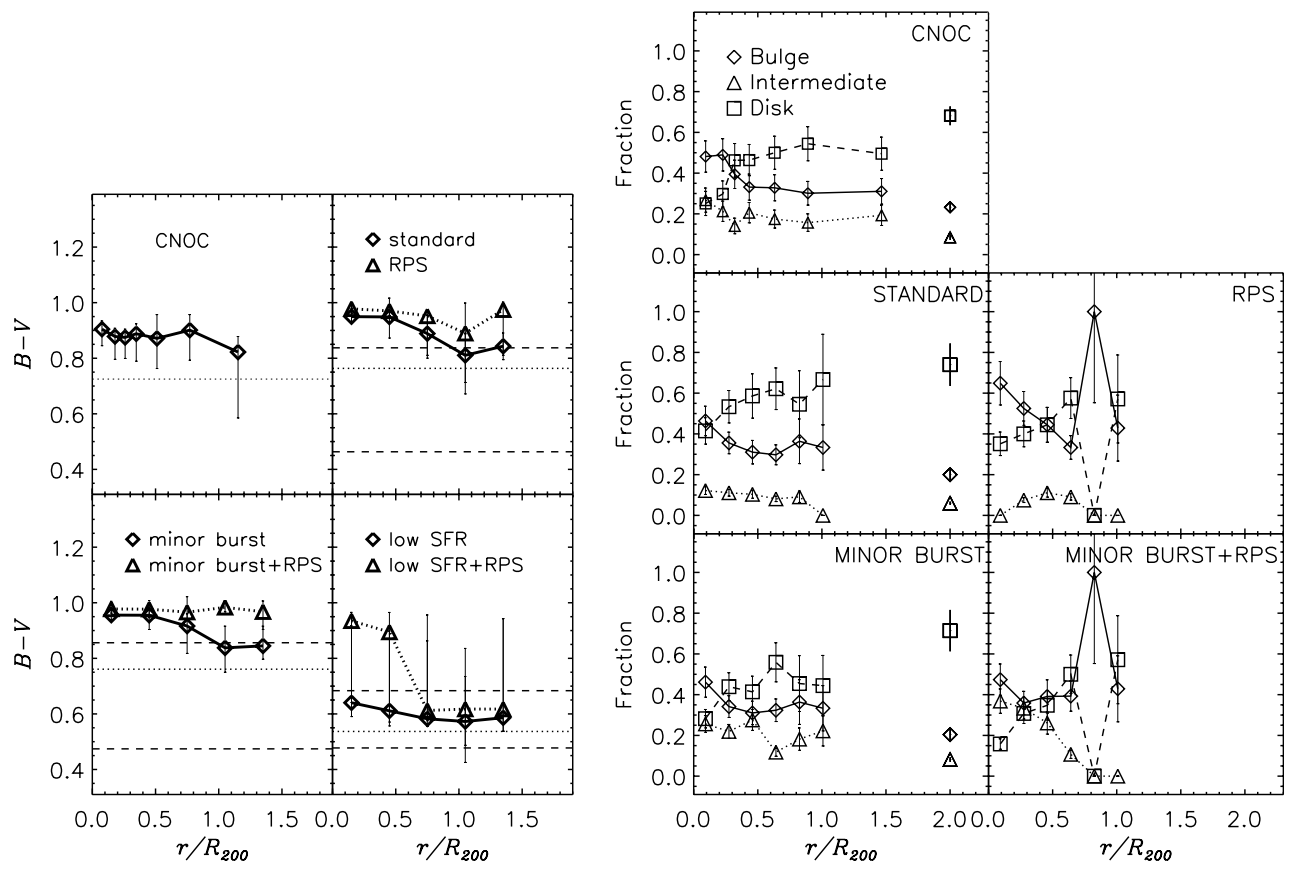

Figure 2. Left: The median $B-V$ colours of cluster galaxies with $M_{R}<-20.5$ are plotted as a function of projected radius. In the upper left panel, the diamonds denote the observed median colours of the CNOC sample. The median colour of field galaxies at the same redshift range are shown by the dotted line. Other panels shows the median colours for the simulated galaxies at $z=0.2$ and the corresponding RPS model is indicated by the triangles in each panel. The field model is also shown by the dotted line in each panel. Right: The morphological fractions are plotted as a function of projected radius. In the top panel we show the observed fractions for the galaxies with $M_{R}<-20.5$ in the CNOC sample. Other panels show the fractions given by our models for the simulated cluster at $z=0.2$. The symbols at $r / R_{200}=2$ indicate the fractions in the field in each panel.

change their $B / T$ by the fading of their disks. In the minor burst + RPS model, we can see the increasing intermediate fraction toward the centre.

\section{Summary}

We have investigated the effects of major mergers, strangulation, RPS of cold disk gas, and minor mergers on the evolution of bright cluster galaxies. We have used a combination of the cosmological $N$-body simulations and the SA galaxy formation model. This method enables us to study the above environmental effects in a fully cosmological context. We have determined the model parameters of the reference model to reproduce galaxy properties at $z=0$.

Our results are summarised as follows.

(a) The process that terminates SF in early-type galaxies $(B / T>0.4)$ in all environments is starburst triggered by a major merger.

(b) If we adopt appropriate SF time-scales, so as to reproduce the observed cold gas mass fraction in the field, the dominant process that determines colours of galaxies in the cluster core is the strangulation. 
(c) Since the strangulation sufficiently suppresses SF in cluster cores, the effect of the RPS is hardly observed.

(d) Minor burst does not affect galaxy properties except for morphology.

(e) Without minor bursts the fraction of intermediate $B / T$ galaxies in clusters becomes too small. The model with the minor burst can reproduce the observed morphology-radius relations. We conclude that the minor burst is an essential process to form intermediate $B / T$ galaxies.

$(f)$ The RPS hardly increases the intermediate population in the cluster without minor bursts. When the minor burst is taken into account, the RPS increases the intermediate galaxy fraction in the cluster cores.

\section{References}

Barnes, J. E. 1996 In ASP Conference Series 92, Formation of the Galactic Halo-Inside and Out, eds. H. L. Morrison \& A. Salajedini (San Francisco: ASP), 415

Bower, R. G., Lucy, J. R., \& Ellis, R. S. 1992, MNRAS, 254, 601

Butcher, H. \& Oemler, A. 1984, ApJ, 285, 426

Carlberg, R. G., Yee, H. K. C., \& Ellingson, E. 1997, ApJ, 478, 462

Diaferio, A., Kauffmann, G., Balogh, M. L., White, S. D. M., Schade, D., \& Ellingson, E. 2001, MNRAS, 323, 999

Dressler A. 1980, ApJ, 236, 351

Dressler A. et al. 1997, ApJ, 490, 577

Gunn, J. E., \& Gott, J. R. 1972, ApJ, 176, 1

Lewis, I., et al. 2002, MNRAS, 334, 673

Okamoto, T. \& Habe, A. 1999, ApJ, 516, 591

Okamoto, T. \& Habe, A. 2000, PASJ, 52, 457

Okamoto, T. \& Nagashima, M. 2001, ApJ, 547, 109

Okamoto, T. \& Nagashima, M. 2003, ApJ, 587, 500

Springel, V., White, S. D. M., Tormen, G., \& Kauffmann, G. 2001, MNRAS, 328, 726

Whitmore, B. C., Gilmore, D. M., \& Jones, C. 1993, ApJ, 407, 489

Yee, H. K. C., Ellingson, E., \& Carlberg, R. G. 1996, ApJS, 102, 269

Walker, I. R., Mihos, J. C., \& Hernquist, L. 1996, ApJ, 460, 121 